\title{
Antiepileptic drug teratogenicity and de novo genetic variation load
}

Piero Perucca, $\mathrm{MD}, \mathrm{PhD}^{1,2,3 *}$, Alison Anderson, $\mathrm{PhD}^{1,2 *}$, Dana Jazayeri, $\mathrm{PhD}^{2}$, Alison Hitchcock, $\mathrm{RN}^{2}$, Janet Graham, $\mathrm{RN}^{2}$, Marian Todaro, $\mathrm{PhD}^{2}$, Torbjörn Tomson, $\mathrm{MD}, \mathrm{PhD}^{4}$, Dina Battino, $\mathrm{MD}^{5}$, Emilio Perucca, $\mathrm{MD}, \mathrm{PhD}^{6}$, Meritxell Martinez Ferri, $\mathrm{MD}^{7}$, Anne Rochtus, $\mathrm{PhD}^{8}$, Lieven Lagae, $\mathrm{MD}, \mathrm{PhD}^{8}$, Maria Paola Canevini, $\mathrm{MD}, \mathrm{PhD}^{9,10}$, Elena Zambrelli, $\mathrm{MD}^{9}$, Ellen Campbell, $\mathrm{MD}^{11}$, Bobby P.C. Koeleman, $\mathrm{PhD}^{12}$, Ingrid E. Scheffer, MBBS, $\mathrm{PhD}^{13,14}$, Samuel F. Berkovic, MD, FRS ${ }^{13}$, Patrick Kwan, FRACP, $\mathrm{PhD}^{1,2,3}$, Sanjay M. Sisodiya, FRCP, $\mathrm{PhD}^{15,16}$, David B. Goldstein, $\mathrm{PhD}^{17}$, Slavé Petrovski, $\mathrm{PhD}^{2,18}$, John Craig, MD ${ }^{11}$, Frank J.E. Vajda, $\mathrm{MD}^{1,2}$, Terence J. O’Brien, $\mathrm{MD}^{1,2,3}$, and the EpiPGX and EPIGEN Consortia

*joint first authors

(1) Department of Neuroscience, Central Clinical School, Monash University;

(2) Departments of Medicine and Neurology, The University of Melbourne, The Royal Melbourne Hospital, Melbourne, Australia;

(3) Department of Neurology, Alfred Health, Melbourne, Australia;

(4) Department of Clinical Neuroscience, Karolinska Institutet, Stockholm, Sweden;

(5) Epilepsy Center, Department of Neurophysiology and Experimental Epileptology,

I.R.C.C.S. Neurological Institute "Carlo Besta" Foundation, Milan, Italy;

(6) Department of Internal Medicine and Therapeutics, University of Pavia, and Clinical Trial Center, IRCCS Mondino Foundation, Pavia, Italy;

(7) Servicio de Neurología, Hospital Mútua de Terrassa, Barcelona, Spain;

(8) Department of Development and Regeneration, Section of Pediatric Neurology, University Hospitals Leuven, Leuven, Belgium;

(9) Child Neuropsychiatry Unit-Epilepsy Center, San Paolo Hospital, Milan, Italy;

(10) Department of Health Sciences, University of Milan, Italy;

(11) Belfast Health and Social Care Trust, Belfast, UK;

(12) Department of Genetics, Center for Molecular Medicine, University Medical Center Utrecht, The Netherlands;

(13) Epilepsy Research Centre, Department of Medicine, Austin Health, The University of Melbourne, Melbourne, Australia;

(14) Department of Paediatrics, The University of Melbourne, Royal Children's Hospital, Florey and Murdoch Children’s Research Institutes, Melbourne, Australia;

(15) Department of Clinical and Experimental Epilepsy, UCL Queen Square Institute of Neurology, London, UK;

(16) Chalfont Centre for Epilepsy, Chalfont-St-Peter, UK;

(17) Institute of Genomic Medicine, Columbia University, New York, USA;

(18) Centre for Genomic Research, AstraZeneca, Cambridge, UK.

Running head: Mechanisms of antiepileptic drug teratogenicity

This is the author manuscript accepted for publication and has undergone full peer review but has not been through the copyediting, typesetting, pagination and proofreading process, which may lead to differences between this version and the Version of Record. Please cite this article as doi: 10.1002/ana.25724

This article is protected by copyright. All rights reserved. 
Characters in Title: 105. Characters in Running head: 54. Words in the Abstract: 250. Words in the Introduction: 438. Words in the Discussion: 741 . Words in the body of the manuscript: 4018. References: 40. Figures: 3. Color figure: 1. Tables: 2. Supplemental tables: 2 .

Addresses for Correspondence: Dr. Piero Perucca, Department of Neurology, The Royal Melbourne Hospital, Parkville, Victoria 3050, Australia. Telephone: +61 3 93428145. Fax: +61 39342 8628. E-mail: piero.perucca@mh.org.au

This article is protected by copyright. All rights reserved. 


\begin{abstract}
Objective: The mechanisms by which antiepileptic drugs (AEDs) cause birth defects (BDs) are unknown. Data suggest that AED-induced BDs may result from a genome-wide increase of de novo variants in the embryo, a mechanism which we investigated.

Methods: Whole-exome sequencing data from child-parent trios were interrogated for de novo single-nucleotide variants/indels (dnSNVs/indels) and copy number variants (dnCNVs). Generalized linear models were applied to assess de novo variant burdens in: children exposed prenatally to AEDs (AED-exposed children) vs children without BDs not exposed prenatally to AEDs (AED-unexposed unaffected children), and AED-exposed children with BDs vs those without BDs, adjusting for confounders. Fisher's exact test was used to compare categorical data.
\end{abstract}

Results: 67 child-parent trios were included: 10 with AED-exposed children with BDs; 46 with AED-exposed unaffected children; 11 with AED-unexposed unaffected children. The dnSNV/indel burden did not differ between AED-exposed children and AED-unexposed unaffected children [median dnSNV/indel number/child (range): 3 (0-7) vs 3 (1-5), p=0.50]. Among AED-exposed children, there were no significant differences between those with BDs and those unaffected. Likely deleterious dnSNVs/indels were detected in 9/67 (13\%) children, none of whom had BDs. The proportion of cases harbouring likely deleterious dnSNVs/indels did not differ significantly between AED-unexposed and AED-exposed children. The dnCNV burden was not associated with AED exposure or birth outcome.

Interpretation: Our study indicates that prenatal AED exposure does not increase the burden of de novo variants, and that this mechanism is not a major contributor to AED-induced BDs. These results can be incorporated in routine patient counselling.

This article is protected by copyright. All rights reserved. 


\section{Introduction}

The broad utilization of antiepileptic drugs (AEDs), not only for the treatment of epilepsy but also for the management of other diseases such as psychiatric disorders, pain and migraine, has resulted in widespread awareness of their adverse effects. ${ }^{1}$ Anatomical teratogenesis, in particular, is a major concern for women of childbearing age on AED therapy. ${ }^{2}$ Prenatal exposure to AEDs is associated with an overall two- to three-fold increased risk of birth defects. ${ }^{3}$ The risk varies across different AED monotherapies, being greatest for valproate (VPA), and is dose-dependent, at least for some AEDs. ${ }^{4,5}$ For pregnancies exposed to polytherapy, the teratogenic risk also depends on the specific drugs included in the combination, particularly VPA, rather than simply the number of AEDs. ${ }^{6,7}$ The wide range of birth defects associated with prenatal AED exposure includes cardiac malformations, neural tube defects, cleft lip/palate, hypospadias, and polydactyly. ${ }^{3}$ Although certain malformations are more common with specific AEDs, e.g. neural tube defects with VPA, ${ }^{8}$ considerable phenotypic overlap exists across different drugs. 9, $10^{10}$

The mechanisms underlying AED-associated birth defects are poorly understood. Experimental and clinical data, including the observation that only a minority of AED-exposed pregnancies result in birth defects, suggest that genetic factors might be implicated. ${ }^{4,11-13}$ Elucidating these factors would be valuable for improved counselling of AED-treated women planning a pregnancy, as well as families in which AED-induced birth defects have occurred. Identification of genetic risk factors could also inform AED selection, and lead to the development of less teratogenic compounds.

The considerable overlap in the types of birth defects across different AEDs suggests that AEDassociated anatomical teratogenesis may arise from dysregulation of one or more shared biological mechanisms. De novo variants are an important mechanism of human disease, including developmental disorders, and investigation of their pathogenic role has been facilitated by use of trio-based next-generation sequencing techniques. ${ }^{14}$ Mutations can arise from exposure to certain drugs, e.g. anticancer agents and immunosuppressants. ${ }^{15,16}$ Several teratogens have mutagenic effects, ${ }^{17}$ and drug-induced mutations have been proposed as a 
mechanism of teratogenesis. ${ }^{17-19}$ The large variety of different types of birth defects seen in babies exposed to AEDs in utero, would be consistent with the hypothesis of AEDs producing 'random' mutations in the genome. The same hypothesis would be consistent with the concept of genetic heterogeneity, where mutations in different genes, including genes implicated in different pathways, can result in the same phenotype. Here, we performed a whole-exome sequencing (WES) trio-based study to test whether exposure in utero to AEDs as a class can lead to increased genome-wide generation of de novo variants, destabilizing the embryonic genome and thus acting as a common mechanism for AED-associated birth defects. 


\section{Methods}

\section{Subjects}

We established a large international collaboration to investigate the molecular underpinnings of AED-associated birth defects, involving centres in Australia, Europe and North America, the Australian Pregnancy Register (APR), and the UK and Ireland Epilepsy and Pregnancy Register. This study is the first endeavour of this collaboration, and is based on cases recruited through the APR. The APR was set up in 1999, and includes prospective or retrospective pregnancies from: a) women with epilepsy on AEDs; b) women with epilepsy not taking AEDs; and c) women taking AEDs for non-epilepsy indications. ${ }^{5,13}$

Between April 2013 and September 2016, we recruited three types of child-parent trios: 1) trios including a child with $\geq 1$ birth defect who was exposed to AEDs in utero (AED-exposed affected child); 2) trios including a child without birth defects who was exposed to AEDs in utero (AED-exposed unaffected child); and 3) trios including a child without birth defects who was not exposed to AEDs in utero (AED-unexposed unaffected child). For the purpose of this study, 'exposure' refers to treatment(s) taken throughout the first trimester of pregnancy, and 'dose' refers to dose taken at time of conception.

The study was approved by the Melbourne Health Human Research and Ethics Committee. Written informed consent was obtained from all participants, or from parents in the case of minors.

\section{Sample collection}

All participants provided blood or saliva samples for genomic DNA extraction. DNA was extracted from blood samples using the Gentra Puregene kit, and from saliva samples using the PSP SlamaGene Module 2 extraction kit. DNA samples of 30-1500 ng/ul meeting a quality threshold of $\sim 1.8$ as determined by the NanoDrop 2000 UV-Vis Spectrophotometer (Thermo Fisher Scientific, Waltham, USA), were selected for sequencing. 


\section{Sequencing and bioinformatics pipeline}

For 143 participants, DNA samples were whole-exome sequenced at the Institute for Genomic Medicine, Columbia University, New York, USA. DNA libraries were constructed using the Kapa Library Preparation Kit Illumina platforms (Kapa Biosystems, Wilmington, USA). For exome capture, SeqCap EZ Exome Enrichment Kit version 3.0 (Roche NimbleGen, Madison, USA) was used according to the manufacturer's protocol. Whole-exome sequencing (WES) was performed on the Illumina HiSeq 2500 platform (Illumina, San Diego, USA), with an average of 100x coverage of 100-bp paired-end reads. After quality filtering the raw sequenced data with CASAVA, the Illumina lane-level FASTQ files were aligned to the Human Reference Genome (Build 37/hg19) using the Barrows-Wheeler Alignment Tool version 0.5.10. Picard (http://broadinstitute.github.io/picard/) was applied to mark duplicate reads and process lanelevel SAM files, resulting in a sample-level BAM file which was used for variant calling.

DNA samples from six additional participants were whole-exome sequenced at the Broad Institute, Cambridge, USA, as part of the Epi25 Collaborative (www.epi-25.org). These samples were sequenced on the Illumina HiSeq X platform. Exome capture was performed with Illumina Nextera Rapid Capture Exomes or TruSeq Rapid Exome enrichment kit. Raw reads were processed using the Picard data-processing pipeline with alignment to Build 37/hg19. Resulting BAM files were then used for variant calling.

Identification of de novo single nucleotide variants and small insertions and deletions (dnSNVs/indels)

Single nucleotide variants (SNVs) and small insertions and deletions (indels) were called for each individual using the Genome Analysis Toolkit (GATK) HaplotypeCaller in GVCF mode, yielding gVCF files. For each family, joint-genotyping, using the gVCFs for each member, was undertaken to generate family-level variant call sets. Variant quality scores for each family call 
set were adjusted using the variant quality score recalibration (VQSR) method, with truth tranche scores between 99.9 and 100 considered to be false positives. For SNVs, genotype quality (GQ) scores were further refined using the GATK genotype refinement pipeline (www. software.broadinstitute.org/gatk/documentation/article?id=11074). This pipeline uses genotype likelihoods derived from ethnic-specific subgroups of the 1000 Genomes project and pedigree information to refine estimates of genotype likelihoods and update GQ scores. This method was applied to 35 families with common ethnicity (33 of European decent and two of South-East Asian decent); for three families with parents from differing ethnic backgrounds, only pedigree information was applied. The GATK VariantAnnotator tool flags putative de novo variants based on sample GQ scores and allele count information. Variant calls are flagged as high-confidence putative variants, where all members of a child-parent trio have a GQ >20 (indicating a 99\% chance that the genotype is correct), or as low-confidence putative variants, where both parents had a GQ $>20$ and the child a GQ $\geq 10$.

High-confidence putative dnSNVs/indels were selected and then filtered to remove those that failed $\geq 1$ site-specific GATK metrics as follows. For SNVs: a quality by depth (QD) score of $<2$; a Fisher Strand (FS) score $>60$ or a symmetric odds ratio (SOR) $>3$, both of which are an indication of strand bias; Root Mean Square of mapping quality (MQ) <40; mapping quality rank sum test (MQRankSum), to detect differences in mapping quality between reads supporting the reference and those supporting an alternative allele, less than -12.5; and MannWhitney Rank Sum Test (ReadPosRankSum), to check for alternative allele reads that are predominately near the end of the read and thus likely errors, of less than -8.0. For indels, reads with a QD $<2$, FS $>200$, ReadPosRankSum less than -20.0 , or SOR of $\geq 3$ were removed. The following criteria were then applied: alternative allele frequency between $30 \%$ and $70 \%$ for heterozygous variants observed in the child (no qualifying homozygous variants were detected) and not present in either parent; and a minimum read depth across all trio members of 15 for SNVs and 30 for indels, which are more technically challenging to call correctly. ${ }^{20}$ Variants observed in child exomes from different families were considered an indication of sequencing artifacts at problematic loci and were therefore removed. DnSNVs/indels shared by $\geq 1$ sibling were also excluded; even if true, these variants are potentially inherited ${ }^{21}$ and unlikely to be 
due to AED-exposure. The remaining variants were screened using the Integrative Genomics Viewer (IGV) to identify and remove those arising from sequencing artefacts and those where a trace of the variant was evident in a parental genome. Those that passed screening were termed 'qualifying dnSNVs/indels', and used to address the study endpoints.

Qualifying dnSNVs/indels were annotated using Ensembl's Variant Effect Predictor (VEP). ${ }^{22}$ Variants were considered likely deleterious if they met the following criteria: impact predicted to be 'HIGH' (probably causing protein truncation, loss of function or triggering nonsensemediated decay) or 'MODERATE' (likely to change protein effectiveness); SIFT classification of 'deleterious' and a PolyPhen classification of 'probably damaging/possibly damaging'; and not observed in the publicly available 1000 genomes (http://www.internationalgenome.org/) and gnomAD (http://gnomad.broadinstitute.org/) databases.

\section{Identification of de novo copy number variants (dnCNVs)}

In addition to dnSNVs/indels, we investigated dnCNVs in child-parent trios. Copy number variant (CNV) calling and genotyping across all samples was performed using the eXome Hidden Markov Model (XHMM) ${ }^{23}$ version 1.0. CNVs were filtered to include only those with a genotyping quality score of $\geq 70$ and with coverage of three or more exome target regions. CNVs that overlapped $50 \%$ of another CNV that occurred in $>10 \%$ of all samples were removed. CNVs detected in a child and not present in the child's parents (dnCNVs) were then called using Plink/Seq version 0.10. Putative dnCNVs observed in more than one family were removed as these likely represent common variation or technical artefacts.

\section{Statistical analysis}

To test the study's primary hypothesis that exposure to AEDs in utero can result in increased genome-wide generation of de novo variants, we applied the generalized linear model (GLM) to compare all qualifying dnSNVs/indels (dnSNV/indel burden) between: a) children exposed

This article is protected by copyright. All rights reserved. 
to AEDs in utero (AED-exposed children) and children without birth defects who were not exposed to AEDs in utero (AED-unexposed unaffected children); b) AED-exposed children with birth defects (AED-exposed affected children) and AED-exposed children without birth defects (AED-exposed unaffected children). These analyses were performed before and after adjustment for potential confounders, i.e. sex and parental age at child's birth. Since no prior data were available for power and sample size calculations, we performed a sensitivity analysis using $\mathrm{G}^{*}$ Power 3.1.9.2 $2^{24}$ to estimate the minimal effect size detectable in the two comparative analyses outlined above.

In secondary analyses, GLM was used to compare the dnSNV/indel burden between: a) children exposed to VPA in utero (VPA-exposed children) and children exposed to other AEDs or not exposed to any AEDs in utero, before and after adjusting for sex and paternal age at child's birth; b) VPA-exposed children with birth defects and VPA-exposed children without birth defects, unadjusted due to small sample size. The above-described comparative analyses were also applied to dnCNVs, to assess between-group differences in all dnCNVs (dnCNV burden).

Fisher's exact test was used to compare the proportion of subjects harbouring likely deleterious dnSNVs/indels between: a) AED-exposed children and AED-unexposed unaffected children: b) VPA-exposed children and children exposed to other AEDs or not exposed to any AEDs in utero. Fisher's exact test was also used to compare other categorical data. Kendall's tau-b correlation coefficient $\left(\tau_{\mathrm{B}}\right)$ was used to test the association between parental age and dnSNVs/indel burden and between parental age and dnCNV burden.

To test for potential dose-dependency in the occurrence of de novo variants, drug loads were determined for each AED-exposed child. Drug loads were computed as the sum of the prescribed daily dose/defined daily dose (DDD) ratios for each AED included in the mother's treatment regimen, where DDD is the assumed average maintenance daily dose of a drug used for its main indication. ${ }^{25}$ Kendall's tau-b correlation coefficient was applied to assess the relationship between drug load and the dnSNV/indel burden, and between drug load and the dnCNV burden. 
All statistical analyses were conducted using $\mathrm{R}$ version 3.4.1.

This article is protected by copyright. All rights reserved. 


\section{Results}

\section{Subject characteristics}

A total of 67 unique child-parent trios (corresponding to 149 individuals from 38 different families) were included in the analysis: 10 comprising an AED-exposed affected child; 46 comprising an AED-exposed unaffected child; and 11 comprising an AED-unexposed unaffected child. Of the 67 children, 38 were males and 29 were females. At the time of the child's birth, median maternal age was 31 (range 19-41) years and median paternal age was 33 (range: 24-43) years. Maternal diagnosis was epilepsy for 65 children, and trigeminal neuralgia for 2 children.

The characteristics of the 10 AED-exposed affected children are provided in Table 1. Six were exposed to AED monotherapy (three to carbamazepine; two to VPA; one to levetiracetam) and four to AED polytherapy (which included VPA in three cases).

Of the 46 AED-exposed unaffected children, 32 were exposed to AED monotherapy and 14 to AED polytherapy. Levetiracetam was the most common monotherapy ( $n=16)$, followed by VPA $(n=5)$, carbamazepine $(n=5)$, lamotrigine $(n=3)$, and oxcarbazepine, phenytoin and topiramate (each $\mathrm{n}=1$ ). Levetiracetam and lamotrigine were the most common AEDs in polytherapy ( $\mathrm{n}=6$ for each), followed by topiramate $(n=5)$, valproate $(n=5)$, carbamazepine $(n=3)$, clonazepam $(n=3)$, lacosamide $(n=2)$, oxcarbazepine and pregabalin (each $n=1$; Table 2).

\section{Qualifying dnSNVs/indels}

A total of 184 qualifying dnSNVs/indels were detected, comprising 175 SNVs, enriched in $\mathrm{G}>\mathrm{A}(\mathrm{n}=44,25.1 \%)$ and $\mathrm{C}>\mathrm{T}(\mathrm{n}=42,24.0 \%)$ transition mutations, and 9 indels. Most ( $\mathrm{n}=139$, 75.5\%) fell within non-protein coding regions. Given the aim of our analysis, these non-coding variants were not excluded, increasing the average number of dnSNVs/indels per exome (2.74) above that generally reported in the literature. ${ }^{26}$ The proportion of dnSNVs/indels within highly 
mutable CpG regions was not significantly different from that reported in a large trio study of families with autism spectrum disorder ${ }^{27}$ [14/184 (0.08\%) vs 108/1692 (0.06\%); p=0.53].

The number of qualifying dnSNVs/indels detected per child ranged from 0 to 7 (median 3), and was significantly higher among children with availability of WES data for siblings $(n=51)$ than those without sibling data $(n=16)$ [median (range): $3(0-7)$ vs $1(0-7) ; p=0.003$ ]. This difference can be attributed to higher GQ scores in exomes informed by sibling data compared to those not informed by sibling data [median (range): 51(0-99) vs 3 (0-99); $\mathrm{p}<0.001$ ]. A higher proportion of unfiltered SNVs met the cut-off limit of a GQ $\geq 20$ in sibling-informed exomes compared to those which were not informed by sibling data [5,990,429/11,018,359 (54\%) vs 3,040,363/8,627,034 (35\%); $<<0.001]$. However, the proportion of children with availability of sibling WES data did not differ between AED-exposed affected children and AED-exposed unaffected children [7/10 (70.0\%) vs 34/46 (73.9\%); p=0.69] or between all AED-exposed children and AED-unexposed unaffected children [41/56 (73.2\%) vs 10/11 (90.9\%); p=0.27). Both paternal and maternal age were correlated with the number of dnSNVs/indels in the offspring (paternal age: $\tau_{\mathrm{B}}=0.28, \mathrm{p}=0.002$; maternal age: $\tau_{\mathrm{B}}=0.23, \mathrm{p}=0.011$; Fig. 1).

\section{DnSNV/indel burden}

The dnSNV/indel burden did not differ between all AED-exposed children and AEDunexposed unaffected children [median number of qualifying dnSNVs/indels per child (range): 3 (0-7) vs 3 (1-5); unadjusted $\mathrm{p}=0.66$, adjusted p=0.50; Fig. 2A]. Among AED-exposed children, no differences were found between children with birth defects and those without birth defects [median number of qualifying dnSNVs/indels per child (range): 3 (0-7) vs 3 (0-7); unadjusted p=0.70, adjusted p=0.90; Fig. 2B]. In a sensitivity analysis, we estimated that our study had an $80 \%$ power to detect at least a 2.44 -fold difference in the dnSNV/indel burden (minimal detectable effect size) between AED-exposed children and AED-unexposed unaffected children at a significance level of 0.05. For the comparison of AED-exposed 
children with birth defects and those without birth defects, the minimal detectable effect size was 2.88.

The dnSNV/indel burden did not differ between VPA-exposed children $(n=15)$ and children either exposed to other AEDs or not exposed to any AEDs in utero $(n=52)$, irrespective of birth outcome [median number of qualifying dnSNVs/indels per child (range): 3 (0-5) vs 3 (0-7), unadjusted $\mathrm{p}=0.83$, adjusted $\mathrm{p}=0.34]$. Among children exposed to VPA in utero either as monotherapy or in combination therapy, no differences were found between children with birth defects $(n=5)$ and those without birth defects $(n=10)$ [median number of qualifying dnSNVs/indels per child (range): 3 (1-4) vs 3 (0-6); $\mathrm{p}=0.69$ ].

AED dose information was available for 51 AED-exposed children (10 with birth defects 41 unaffected). There was no correlation between drug load and the dnSNV/indel burden ( $\tau_{\mathrm{B}}=$ 0.02, p=0.83; Fig 3A).

\section{Likely deleterious dnSNV/indel burden}

A likely deleterious dnSNV/indel was harboured by 9 of the 67 children (13\%). As detailed in Table S1, these were missense variants which were detected in nine different genes: PLCE1, AZU1, KCNH7, MDH1B, ADAMTSL1, OR10Q1, TMEM200C, TRIP13, and FBXO4, none of which is known to be associated with epilepsy.

Notably, none of the children harbouring a likely deleterious dnSNV/indel had birth defects. Only five of these children had been exposed to AEDs in utero (two to VPA monotherapy and three to AED polytherapy; Table S1). The proportion of subjects harbouring likely deleterious dnSNVs/indels did not differ between AED-unexposed and AED-exposed children [4/11 (36.4\%) vs 5/46 (10.9\%), $\mathrm{p}=0.059]$.

\section{DnCNV burden}


Based on exome target read depth values, an AED-exposed unaffected child was found to be an outlier and was removed from further analyses. A total of $27 \mathrm{dnCNVs}$ were detected among the remaining 66 children. The number of dnCNVs per child ranged from 0 to 2 (median 0 ; mean 0.41). Parental age was not associated with the number of dnCNV in the offspring (paternal age $\tau_{\mathrm{B}}=-0.004, \mathrm{p}=0.96$; maternal age $\tau_{\mathrm{B}}=-0.06, \mathrm{p}=0.53$ ). Thus, subsequent analyses were adjusted for sex only.

The dnCNV burden did not differ between AED-exposed children and AED-unexposed unaffected children [median number of dnCNVs/child (range): 0 (0-2) vs 0 (0-2); unadjusted $\mathrm{p}=0.44$, adjusted $\mathrm{p}=0.83]$. Among AED-exposed children, there were no differences between children with birth defects and those without birth defects [median number of dnCNVs/child (range): 0 (0-1) vs 0 (0-2); unadjusted $\mathrm{p}=0.92$, adjusted $\mathrm{p}=0.75$ ].

The dnCNV burden also did not differ between VPA-exposed children and children exposed to other AEDs or no AEDs in utero [median number of dnCNVs per child (range): 0 (0-2) vs 0 (0-2); unadjusted $\mathrm{p}=0.39$, adjusted $\mathrm{p}=0.62]$. Among children exposed to VPA in utero either as monotherapy or in combination therapy, there were no differences between children with birth defects and those without birth defects [median number of dnCNVs per child (range): 0 (0-1) vs 0.5 (0-2); unadjusted $\mathrm{p}=0.24]$.

In the 51 AED-exposed children for whom AED dose information was available, no correlation was found between drug load and the dnCNV burden $\left(\tau_{\mathrm{B}}=-0.09, \mathrm{p}=0.45\right.$; Fig. 3B $)$.

\section{Within family comparisons}

In 17 families, two or more children had been exposed to AEDs in utero. In these families, the difference in the dnSNV/indel burden between siblings ranged from 0 to 5 (median 2). In five families, the AED-exposed affected child and one or more siblings without birth defects had been exposed to the same AED(s): the dnSNV/indel burden was highest in the affected child in $2 / 5$ families, and equivalent to or lower than that of an unaffected sibling in $3 / 5$ families. 
The difference in the dnCNV burden between siblings across the 17 families ranged from 0 to 2 (median 0). In the five families with children exposed to the same AED(s), the dnCNV burden was highest in the affected child in 2/5 families. In one family comprising five children exposed to the same AED regimen, the affected child had a single dnCNV and in the unaffected siblings the number of dnCNVs ranged from 0 to 2. In 2/5 families, no dnCNVs were found. 


\section{Discussion}

More than 50 years since the report by $\mathrm{Meadow}^{28}$ of hare-lip, cleft palate and other congenital abnormalities in children born to mothers taking primidone, phenytoin or phenobarbital, the molecular mechanisms underpinning AED-associated birth defects remain elusive. We conducted a trio-based WES study to investigate for the first time the possibility of AEDassociated birth defects resulting from an increased genome-wide generation of de novo variants in the embryo. This hypothesis was based on three observations: a) congenital malformations develop only in a minority of AED-exposed foetuses; ${ }^{3,4} \mathrm{~b}$ ) there is considerable phenotypic overlap across congenital malformations associated with different AEDs, ${ }^{3,} 10$ suggesting that AED-associated birth defects possibly arise from dysregulation of a shared mechanism or pathway critical to foetal development; and c) several teratogens, including drugs, have genotoxic or mutagenic effects. ${ }^{17}$ Although there is no conclusive evidence for AEDs being mutagenic, chromosomal/DNA damaging effects in vitro or in vivo have been reported for several AEDs, including VPA, ${ }^{29-31}$ phenytoin, ${ }^{29}$, 32, 33 barbiturates, ${ }^{29}$ carbamazepine, ${ }^{29}, 34,35$ oxcarbazepine, ${ }^{36}$ and possibly lamotrigine. ${ }^{30}$ At least for VPA, the suggestion has been made that increased DNA damage may result from chronic inhibition of histone deacetylase. ${ }^{37}$ Despite these premises, we did not find any difference in the dnSNV/indel burden between AED-exposed children and AED-unexposed unaffected children, or between AED-exposed affected children and AED-exposed unaffected children. Likewise, the dnSNV/indel burden did not differ significantly between children exposed to the highly teratogenic agent VPA and children exposed to other AEDs or not exposed to any AEDs, or between VPA-exposed affected children and VPA-exposed unaffected children. Investigation of the dnCNV burden yielded similar findings with no significant differences across the comparison groups. Collectively, these results strongly argue against an AEDinduced increase in the burden of de novo variants being a major contributor to the occurrence of AED-associated birth defects.

We also investigated whether AED-associated birth defects may result from the occurrence of deleterious dnSNVs/indels induced by prenatal AED exposure. None of the AED-exposed 
affected children harboured a likely deleterious dnSNV/indel. The proportion of individuals harbouring likely deleterious dnSNVs/indels did not differ significantly between AEDunexposed and AED-exposed children. Furthermore, there were no differences in the proportion of individuals with a likely deleterious dnSNV/indel between VPA-exposed children and children exposed to other AEDs or not exposed to any AEDs. These findings complement those obtained on the dnSNV/indel and dnCNV burdens, indicating that AEDinduced mutagenesis is unlikely to play an important role in AED teratogenicity.

Our study included 149 subjects from 38 different families. With respect to statistical power considerations, a useful reference of the genetic variation occurring in the general population is provided, for example, by the observation that in fathers the number of de novo variants double every 16.5 years, and increase by eight-fold in 50 years. ${ }^{38}$ We estimated that, for the primary analyses, our study was powered to detect between-group differences of even smaller magnitude (2.44-/2.88-fold). Thus, our results can exclude increased generation of de novo variants as a major mechanism of the anatomical teratogenicity of AEDs as a class. However, larger samples would be needed to assess modest effect sizes or the role of mutagenesis in causing specific AED-associated birth defects. Larger samples would also be required to determine whether mutagenesis is implicated in the teratogenicity associated with specific AEDs, including VPA for which our analyses were limited by small numbers. It should be emphasised that trio-based genetic investigations of AED-associated birth defects are difficult to undertake because these defects are relatively rare and parents, particularly mothers, can be reluctant to engage in genetic investigations which could amplify harboured feelings of guilt and do not provide any immediate benefit to their affected child. These challenges may explain why no prior study has been performed to explore the role to de novo variants in AEDassociated birth defects.

This study represents a first step towards elucidating genetic mechanisms of AED-associated birth defects. Other mechanisms that need to be investigated include the potential role of somatic mutations and epigenetic modifications; the latter may be particularly relevant for VPA, which is a histone deacetylase inhibitor. ${ }^{39}$ Future studies should also attempt to unmask the genetic predisposition to AED anatomical teratogenesis which has been suggested by 
different lines of evidence, including the increased risk of AED-associated birth defects in the presence of a family history of congenital malformations ${ }^{40}$ or a history of foetal malformations in a previous AED-exposed offspring. ${ }^{13}$ Advances are much awaited, and our newly established international collaboration carries the promise of furthering the field.

This article is protected by copyright. All rights reserved. 


\section{Acknowledgements}

The study was funded by a National Health and Medical Research Council (NHMRC) Project Grant (APP1059858) to T.J.O’B., S.P., D.B.G., F.J.E.V., P.K., J.C., S.F.B. and I.E.S.; an NHMRC Program Grant (APP1091593) to T.J.O’B., S.F.B. and I.E.S.; and the European Commission grant 279062, EpiPGX (to EpiPGX consortium, B.P.C.K., J.C. and S.M.S.). P.P. is supported by an NHMRC Early Career Fellowship (APP1163708), and by the Viertel Clinical Investigator Award from the Sylvia and Charles Viertel Charitable Foundation. A.R. is funded by internal funds of the University of Leuven (PDM/17/195).

The authors are grateful to all individuals who took part in the study. They are also thankful to Dr Zhibin Chen for his advice on statistical analysis.

\section{Author contributions}

P.P., A.A., I.E.S., S.F.B., P.K., D.B.G., S.P., J.C., F.J.E.V. and T.J.O’B contributed to the concept and study design. All authors contributed to data acquisition and analysis. P.P., A.A., T.J.O’B drafted the manuscript and figures, and all authors approved the final version.

Members of the EpiPGX and EPIGEN consortia are listed in Table S2.

\section{Potential conflicts of interests}

Nothing to report. 


\section{References}

1. Perucca P, Gilliam FG. Adverse effects of antiepileptic drugs. Lancet Neurol. 2012 Sep;11(9):792-802.

2. Thomas RH, Hammond CL, Bodger OG, et al. Identifying and prioritising epilepsy treatment uncertainties. J Neurol Neurosurg Psychiatry. 2010 Aug;81(8):918-21.

3. Tomson T, Battino D. Teratogenic effects of antiepileptic drugs. Lancet Neurol. 2012 Sep;11(9):803-13.

4. Tomson T, Battino D, Bonizzoni E, et al. Comparative risk of major congenital malformations with eight different antiepileptic drugs: a prospective cohort study of the EURAP registry. Lancet Neurology. 2018 Jun;17(6):530-8.

5. Vajda FJ, O'Brien TJ, Graham JE, Lander CM, Eadie MJ. Dose dependence of fetal malformations associated with valproate. Neurology. 2013 Sep 10;81(11):999-1003.

6. Vajda FJ, Hitchcock AA, Graham J, O'Brien TJ, Lander CM, Eadie MJ. The teratogenic risk of antiepileptic drug polytherapy. Epilepsia. 2010 May;51(5):805-10.

7. Tomson T, Battino D, Bonizzoni E, et al. Dose-dependent teratogenicity of valproate in mono- and polytherapy: an observational study. Neurology. 2015 Sep 8;85(10):866-72.

8. Jentink J, Loane MA, Dolk H, et al. Valproic acid monotherapy in pregnancy and major congenital malformations. N Engl J Med. 2010 Jun 10;362(23):2185-93.

9. Veroniki AA, Cogo E, Rios P, et al. Comparative safety of anti-epileptic drugs during pregnancy: a systematic review and network meta-analysis of congenital malformations and prenatal outcomes. BMC Med. 2017 May;15:20.

10. Tomson T, Battino D, Perucca E. Valproic acid after five decades of use in epilepsy: time to reconsider the indications of a time-honoured drug. Lancet Neurol. 2016 Feb;15(2):210-8.

11. Duncan S, Mercho S, Lopes-Cendes I, et al. Repeated neural tube defects and valproate monotherapy suggest a pharmacogenetic abnormality. Epilepsia. 2001 Jun;42(6):750-3.

12. Finnell RH, Gould A, Spiegelstein O. Pathobiology and genetics of neural tube defects. Epilepsia. 2003;44 Suppl 3:14-23.

This article is protected by copyright. All rights reserved. 
13. Vajda FJ, O'Brien TJ, Lander CM, Graham J, Roten A, Eadie MJ. Teratogenesis in repeated pregnancies in antiepileptic drug-treated women. Epilepsia. 2013 Jan;54(1):181-6.

14. Acuna-Hidalgo R, Veltman JA, Hoischen A. New insights into the generation and role of de novo mutations in health and disease. Genome Biol. 2016 Nov 28;17(1):241.

15. Szikriszt B, Poti A, Pipek O, et al. A comprehensive survey of the mutagenic impact of common cancer cytotoxics. Genome Biol. 2016 May;17:16.

16. Ciliao HL, Camargo-Godoy RBD, Barcelos GRM, Zanuto A, Delfino VDA, Colus IMD. Long-term genotoxic effects of immunosuppressive drugs on lymphocytes of kidney transplant recipients. Mutation Research-Genetic Toxicology and Environmental Mutagenesis. 2016 Aug;806:47-52.

17. Rencuzogullari E, Aydin M. Genotoxic and mutagenic studies of teratogens in developing rat and mouse. Drug Chem Toxicol. 2018 May 10:1-21.

18. Wilson JG. Environment and Birth Defects. New York: Academic Press; 1973.

19. Finnell RH. Teratology: general considerations and principles. J Allergy Clin Immunol. 1999 Feb;103(2 Pt 2):S337-42.

20. De Summa S, Malerba G, Pinto R, Mori A, Mijatovic V, Tommasi S. GATK hard filtering: tunable parameters to improve variant calling for next generation sequencing targeted gene panel data. BMC Bioinformatics. 2017 Mar 23;18(Suppl 5):119.

21. Jonsson H, Sulem P, Arnadottir GA, et al. Multiple transmissions of de novo mutations in families. Nat Genet. 2018 Dec;50(12):1674-80.

22. McLaren W, Gil L, Hunt SE, et al. The Ensembl Variant Effect Predictor. Genome Biol. 2016 Jun 6;17(1):122.

23. Fromer M, Purcell SM. Using XHMM Software to Detect Copy Number Variation in Whole-Exome Sequencing Data. Curr Protoc Hum Genet. 2014 Apr 24;81:7 23 1-1.

24. Faul F, Erdfelder E, Buchner A, Lang AG. Statistical power analyses using G*Power 3.1: tests for correlation and regression analyses. Behav Res Methods. 2009 Nov;41(4):114960.

25. World Health Organization Collaborating Centre for Drug Statistics Methodology. Purpose of the ATC/DDD system. [14 January 2020]; Available from: https://www.whocc.no/atc_ddd_index/?code=N03A. 
26. Wilfert AB, Sulovari A, Turner TN, Coe BP, Eichler EE. Recurrent de novo mutations in neurodevelopmental disorders: properties and clinical implications. Genome Med. 2017 Nov 27;9(1):101.

27. Iossifov I, O'Roak BJ, Sanders SJ, et al. The contribution of de novo coding mutations to autism spectrum disorder. Nature. 2014 Nov;515(7526):216-U136.

28. Meadow SR. Anticonvulsant drugs and congenital abnormalities. Lancet. 1968 Dec 14;2(7581):1296.

29. Curatolo P, Brinchi V, Cusmai R, Vignetti P, Benedetti P. Increased chromosomal breakage in epileptic children after long-term treatment. Eur J Pediatr. 1986 Oct;145(5):43942.

30. Fucic A, Stojkovic R, Miskov S, et al. Transplacental genotoxicity of antiepileptic drugs: animal model and pilot study on mother/newborn cohort. Reprod Toxicol. 2010 Dec;30(4):613-8.

31. Khan S, Ahmad T, Parekh CV, Trivedi PP, Kushwaha S, Jena G. Investigation on sodium valproate induced germ cell damage, oxidative stress and genotoxicity in male Swiss mice. Reprod Toxicol. 2011 Dec;32(4):385-94.

32. Morel CF, Duncan AM, Desilets V. A fragile site at 10q23 (FRA10A) in a phenytoinexposed fetus: a case report and review of the literature. Prenat Diagn. 2005 Apr;25(4):318-21. 33. Herha J, Obe G. Chromosomal damage in epileptics on monotherapy with carbamazepine and diphenylhydantoin. Hum Genet. 1976 Dec 15;34(3):255-63.

34. Awara WM, El-Gohary M, El-Nabi SH, Fadel WA. In vivo and in vitro evaluation of the mutagenic potential of carbamazepine: does melatonin have anti-mutagenic activity? Toxicology. 1998 Jan 16;125(1):45-52.

35. Celik A. The assessment of genotoxicity of carbamazepine using cytokinesis-block (CB) micronucleus assay in cultured human blood lymphocytes. Drug Chem Toxicol. 2006;29(2):227-36.

36. Akbar H, Khan A, Mohammadzai I, Khisroon M, Begum I. The genotoxic effect of oxcarbazepine on mice blood lymphocytes. Drug Chem Toxicol. 2018 Apr;41(2):135-40. 
37. Coyle TE, Bair AK, Stein C, Vajpayee N, Mehdi S, Wright J. Acute leukemia associated with valproic acid treatment: a novel mechanism for leukemogenesis? Am J Hematol. 2005 Apr;78(4):256-60.

38. Kong A, Frigge ML, Masson G, et al. Rate of de novo mutations and the importance of father's age to disease risk. Nature. 2012 Aug 23;488(7412):471-5.

39. Phiel CJ, Zhang F, Huang EY, Guenther MG, Lazar MA, Klein PS. Histone deacetylase is a direct target of valproic acid, a potent anticonvulsant, mood stabilizer, and teratogen. $\mathrm{J}$ Biol Chem. 2001 Sep 28;276(39):36734-41.

40. Tomson T, Battino D, Bonizzoni E, et al. Dose-dependent risk of malformations with antiepileptic drugs: an analysis of data from the EURAP epilepsy and pregnancy registry. Lancet Neurology. 2011 Jul;10(7):609-17. 


\section{Figures}

Figure 1. Correlation between parental age and dnSNV/indel burden. (A) Paternal age and dnSNV/indel burden. (B) Maternal age and dnSNV/indel burden.

Figure 2. DnSNV/indel burden in AED-exposed children vs AED-unexposed unaffected children (A) and in AED-exposed affected children vs AED-exposed unaffected children (B).

Figure 3. Correlation between drug load and de novo variant burdens. (A) Drug load and dnSNV/indel burden. (B) Drug load and dnCNV burden. 


\section{Tables}

Table 1. Characteristics of the 10 AED-exposed affected children.

\begin{tabular}{|c|c|c|c|c|c|c|}
\hline Child & Sex & Birth defects & $\begin{array}{l}\text { AEDs in utero } \\
\text { (mg/day) }\end{array}$ & $\begin{array}{l}\text { Maternal } \\
\text { diagnosis }\end{array}$ & $\begin{array}{c}\text { Family } \\
\text { history of } \\
\text { birth defects }\end{array}$ & $\begin{array}{c}\text { Folic acid } \\
\text { before and } \\
\text { during } \\
\text { pregnancy } \\
\text { (mg/day) }\end{array}$ \\
\hline EA1 & $\mathrm{F}$ & $\begin{array}{l}\text { Arnold-Chiari malformation, } \\
\text { clinodactyly, cardiac defects }\end{array}$ & VPA (1500) & $\begin{array}{l}\text { Generalised } \\
\text { epilepsy }\end{array}$ & No & Yes (5) \\
\hline EA2 & $\mathrm{F}$ & $\begin{array}{l}\text { Thumb hypoplasia, } \\
\text { syndactyly, cardiac defects }\end{array}$ & VPA (1000) & Focal epilepsy & No & Yes (5) \\
\hline EA3 & M & Clubfoot & $\begin{array}{l}\text { VPA (1000), LEV } \\
(1500)\end{array}$ & Focal epilepsy & No & $\mathrm{n} / \mathrm{a}$ \\
\hline EA4 & M & Hypospadias & $\begin{array}{l}\text { VPA (800), CLN } \\
(0.25)\end{array}$ & $\begin{array}{l}\text { Generalised } \\
\text { epilepsy }\end{array}$ & No & Yes (5) \\
\hline EA5 & M & $\begin{array}{l}\text { Macrocephaly, facial } \\
\text { dysmorphic features and } \\
\text { ankyloglossia }\end{array}$ & CBZ (1200) & Focal epilepsy & $\begin{array}{l}\text { Yes (paternal } \\
\text { niece with } \\
\text { spina bifida) }\end{array}$ & Yes (5) \\
\hline EA6 & M & Genital defects & CBZ (500) & Focal epilepsy & No & Yes (5) \\
\hline EA7 & M & Toe aplasia and syndactyly & CBZ (400) & $\begin{array}{l}\text { Generalised } \\
\text { epilepsy }\end{array}$ & No & Yes (5) \\
\hline EA8 & M & Cryptorchidism & $\begin{array}{l}\text { PGB (450), TPM } \\
(300)\end{array}$ & Focal epilepsy & No & Yes (5) \\
\hline EA9 & M & Toe aplasia and syndactyly & LEV (1000) & $\begin{array}{l}\text { Generalised } \\
\text { epilepsy }\end{array}$ & $\begin{array}{c}\text { Yes (mother } \\
\text { with cardiac } \\
\text { abnormalities) }\end{array}$ & Yes (5) \\
\hline EA10 & $\mathrm{F}$ & $\begin{array}{l}\text { Ventricular septal defects } \\
\text { (bulbus cordis and cardiac } \\
\text { septal closure) }\end{array}$ & $\begin{array}{l}\text { VPA (1000), LTG } \\
(200)\end{array}$ & $\begin{array}{l}\text { Generalised } \\
\text { epilepsy }\end{array}$ & No & Yes (5) \\
\hline
\end{tabular}

CBZ=carbamazepine; CLN=clonazepam; F-female; LEV=levetiracetam; LTG=lamotrigine; $\mathrm{M}=$ male; $\mathrm{n} / \mathrm{a}=$ not available; $\mathrm{PGB}=$ pregabalin; $\mathrm{TPM}=$ topiramate; $\mathrm{VPA}=$ =valproic acid 
Table 2. AED polytherapy regimens in AED-exposed unaffected children.

\begin{tabular}{|l|l|}
\hline Child & AEDs in utero (mg/day) \\
\hline EU8 & LCM (200), LTG (300) \\
\hline EU11 & TPM (150), LEV (4000) \\
\hline EU20 & TPM (400), LTG (400) \\
\hline EU21 & TPM (400), LTG (400), LEV (2000) \\
\hline EU22 & LTG (300), LEV (4000) \\
\hline EU25 & VPA (1000), CLN (0.25) \\
\hline EU26 & LTG (dose unknown), LEV (dose unknown) \\
\hline EU27 & LCM (150), TPM (200), CBZ (800) \\
\hline EU30 & LEV (750), OXC (600) \\
\hline EU40 & VPA (700), LTG (200) \\
\hline EU43 & TPM (300), PGB (450) \\
\hline EU44 & VPA (1000), LEV (1500) \\
\hline EU45 & VPA (dose unknown), CBZ (dose unknown), CLN (dose unknown) \\
\hline EU46 & VPA (dose unknown), CBZ (dose unknown), CLN (dose unknown) \\
\hline
\end{tabular}

$\mathrm{CBZ}=$ carbamazepine; $\mathrm{CLN}=$ clonazepam; $\mathrm{LCM}=$ lacosamide; $\mathrm{LEV}=$ levetiracetam; LTG=lamotrigine; OXC=oxcarbazepine; $\mathrm{PGB}=$ pregabalin; TPM=topiramate; VPA=valproic acid

This article is protected by copyright. All rights reserved. 


\section{University Library}

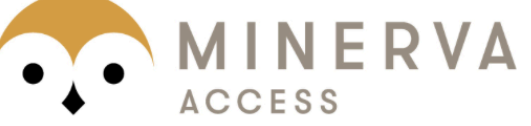

A gateway to Melbourne's research publications

Minerva Access is the Institutional Repository of The University of Melbourne

Author/s:

Perucca, P;Anderson, A;Jazayeri, D;Hitchcock, A;Graham, J;Todaro, M;Tomson, T;Battino, D;Perucca, E;Ferri, MM;Rochtus, A;Lagae, L;Canevini, MP;Zambrelli, E;Campbell,

E;Koeleman, BPC;Scheffer, IE;Berkovic, SF;Kwan, P;Sisodiya, SM;Goldstein, DB;Petrovski, S;Craig, J;Vajda, FJE;O'Brien, TJ

Title:

Antiepileptic Drug Teratogenicity and De Novo Genetic Variation Load

Date:

2020-04-15

Citation:

Perucca, P., Anderson, A., Jazayeri, D., Hitchcock, A., Graham, J., Todaro, M., Tomson, T., Battino, D., Perucca, E., Ferri, M. M., Rochtus, A., Lagae, L., Canevini, M. P., Zambrelli, E., Campbell, E., Koeleman, B. P. C., Scheffer, I. E., Berkovic, S. F., Kwan, P. ,... O'Brien, T. J. (2020). Antiepileptic Drug Teratogenicity and De Novo Genetic Variation Load. ANNALS OF NEUROLOGY, 87 (6), pp.897-906. https://doi.org/10.1002/ana.25724.

Persistent Link:

http://hdl.handle.net/11343/275630 